\title{
Effect of Diabetes Mellitus on Tuberculosis Treatment Outcome and Adverse Reactions in Patients Receiving Directly Observed Treatment Strategy in India: A Prospective Study
}

\author{
Ali Nasir Siddiqui, ${ }^{1}$ Khalid Umer Khayyam, ${ }^{2}$ and Manju Sharma ${ }^{3}$ \\ ${ }^{1}$ Department of Pharmaceutical Medicine, Faculty of Pharmacy, Jamia Hamdard, New Delhi 110062, India \\ ${ }^{2}$ Department of Epidemiology \& Public Health, National Institute of TB and Respiratory Diseases, New Delhi 110030, India \\ ${ }^{3}$ Department of Pharmacology, Faculty of Pharmacy, Jamia Hamdard, New Delhi 110062, India \\ Correspondence should be addressed to Manju Sharma; msharma@jamiahamdard.ac.in
}

Received 21 March 2016; Revised 24 May 2016; Accepted 7 June 2016

Academic Editor: Subash Babu

Copyright ( 2016 Ali Nasir Siddiqui et al. This is an open access article distributed under the Creative Commons Attribution License, which permits unrestricted use, distribution, and reproduction in any medium, provided the original work is properly cited.

\begin{abstract}
Despite successful implementation of directly observed treatment, short course (DOTS) in India, the growing number of diabetes mellitus (DM) patients appears to be a cause in the increasing tuberculosis (TB) incidence, affecting their management. In this regard, a prospective study was conducted on DOTS patients in three primary health care centers in urban slum region of South Delhi, India, to evaluate the effect of DM on sputum conversion, treatment outcome, and adverse drug reactions (ADR) due to anti-TB treatment. Eligible TB patients underwent blood glucose screening at treatment initiation. Disease presentation, clinical outcome, and ADRs were compared between patients of TB with and without DM. Out of 316 patients, the prevalence of DM was found to be $15.8 \%$, in which $19.4 \%$ and $9.6 \%$ were PTB and EPTB patients, respectively. DM patients have observed higher sputum positivity (OR $1.24795 \%$ CI; $0.539-2.886$ ) at the end of 2-month treatment and poor outcome (OR $1.17695 \%$ CI; $0.310-4.457$ ) at the completion of treatment compared with non DM patients. Presence of DM was significantly associated (OR 3.578 95\% CI; $1.114-$ 11.494, $p=0.032$ ) with the development of ADRs. DM influences the treatment outcome of PTB patients in our setting and also on the ADR incidence.
\end{abstract}

\section{Introduction}

The bidirectional association between tuberculosis (TB) and diabetes mellitus (DM) is currently one of the major concerns for clinicians, as DM affects the disease presentation and clinical outcome of TB and vice versa [1]. This comorbidity is known since the beginning of the 20th century. However, recent increase in the number of DM patients, attributed mainly to the modern lifestyle changes, created interest to further assess the association between both diseases [2]. The coepidemic is emerging predominantly in resource poor countries where the burden of DM is increasing and also TB is highly endemic [3].

The prevalence of DM in India is rising and estimated to reach 123.5 million by 2040 [4]. India ranks the highest in TB burden with $23 \%$ of the global incidence cases in
2015 [5]. Active TB and reactivation of latent infection have long been known to be a risk of DM. A recent systematic review demonstrated approximately 3 times higher risk of developing TB in DM patients than no-DM patients [6]. TB infection also deteriorates the glycemic control and reduces the effectiveness of DM management [7]. Multiple studies from different countries reported $12 \%-44 \%$ of TB cases linked with $\mathrm{DM}$ at the time of TB diagnosis [8-12]. The patients of pulmonary TB with DM experienced poor rate of sputum conversion at the end of 2-month regimen along with higher rates of treatment failure and deaths at the end of treatment as compared to no-DM patients [13-15]. Fewer South Indian studies have reported higher DM prevalence but scarce data is available from Northern India $[8,9,11]$.

The anti-TB therapy includes a long-time, wide spectrum of drugs, which can predispose patients to develop adverse 
drug reactions. The emergence of adverse reaction depends on the patients' characteristics and also on concomitant medication during therapy [16]. The use of anti-DM medication may lead to interactions with antitubercular drugs. A subjective assessment is therefore essential to elucidate the factors associated with anti-TB medication adverse reaction, which may determine adherence and, therefore, therapy success.

In this underlying work, we report some of the information gaps that have been recognized on the association between TB and DM, particularly from North India [17]. A systematic assessment is needed as the merging epidemics, especially in low- to middle-income countries, are experiencing the fastest increase in DM prevalence with highest TB burden [18]. Paucity of literatures and lack of awareness increase in the challenge of the management of such patients as the burden of DM is uninterruptedly rising. The objective of present study was to describe the disease presentation, sputum conversion, treatment outcomes, and adverse drug reaction (ADR) incidences in patients of TB with and without DM.

\section{Methods}

2.1. Study Population. Three outpatient primary healthcare centers (PHC), namely, Mehrauli, Khanpur, and Tigri, were selected from South Delhi, India, for patient enrollment from January 2014 to September 2014. These PHCs are affiliated to a tertiary institute, that is, National Institute of TB and Respiratory Diseases (NITRD). All PHCs were located in the urban slum part of South Delhi. TB was diagnosed on the basis of clinical presentation and was confirmed by microscopic detection of acid-fast bacilli (AFB).

2.2. Ethical Approval. This study was approved by the ethics committees of Hamdard University and National Institute of TB and Respiratory Diseases (NITRD/EC/2014/10293), New Delhi, India. Written informed consent was obtained from all subjects before the patient enrollment.

2.3. Study Design and Sample Selection. The new and retreatment TB patients above 15 years of age and attending directly observed treatment, short course (DOTS) clinics at selected $\mathrm{PHCs}$ were undertaken in this prospective study. Eligible patients included those of category I (new cases of sputum smear positive, sputum smear negative, extra pulmonary tuberculosis, and other cases) or category II (retreatment cases of recurrent TB, treatment after failure, treatment after loss to follow-up, and other previously treated patients) were considered for this study. Patients below 15 years of age, suspected or known multidrug resistance (MDR) TB patients, and those who were not willing to participate were excluded from the study. In addition, patients diagnosed with any disease other than TB and DM were also excluded to avoid the confounding effect on treatment outcome. In this study, the proportion of TB patient was estimated to be $25 \%$. We calculated sample size at $95 \%$ confidence interval for proportion $p$ with margin of error $d$ according to the formula: $n=(1.96)^{2} p q / d^{2}$, where $q=1-p \cdot(1.96)^{2} \times 0.25 \times$ $0.75 /(0.05)^{2}=288$. With an estimated $10 \%$ loss to follow-up, a total of 316 patients were enrolled in this study from all three centers.

2.4. Measurement of Glucose Concentration. All enrolled patients were screened for fasting blood glucose (FBG) at TB treatment initiation. Those whose FBG was found beyond $110 \mathrm{mg} / \mathrm{dL}$ were repeated for 2-hour plasma glucose (2 h PG) after $75 \mathrm{~g}$ oral glucose tolerance test (OGTT). DM was diagnosed if the $2 \mathrm{~h}$ PG was found $\geq 200 \mathrm{mg} / \mathrm{dL}$ in accordance with international criteria $[19,20]$. Prediabetes patients were not included in DM category. Finally, TB patients were classified into two groups, one with DM and another with no DM, based on their DM status. Newly diagnosed patients were referred to local PHC physician for DM management, and details were recorded in predefined record sheets. The prescription details and ongoing medication were also recorded for known DM patients.

2.5. Collection of Covariates and Other Symptoms. A pretested, semistructured questionnaire was designed to collect information on sociodemographic profiles, clinical presentation, and signs and symptoms at treatment initiation. Additionally, status of DM with their management, previous TB treatment history, treatment results, medications, duration of $\mathrm{DM}$, and outcome of therapy were recorded in standardized data collection sheet. Sign and symptoms were calculated in a score of 1 to 3, with lower numbers reflecting the higher severity of symptom. Presence of cough, weight loss, evening fever, anorexia, dyspnea, chest pain, and hemoptysis was recorded by face-to-face interviews. Patients with a composite score of 07 , one for each symptom, were classified as being highly symptomatic. Disease severity was evaluated by sputum mycobacterial load. Sputum sample of PTB patients was subjected to microscopic examination of Ziehl-Neelsen staining and was performed for acid-fast bacilli [21]. Mycobacterial load in the sputum was graded as ,+++ , or +++ . Patients were followed up for repeat sputum examination at the end of intensive phase (IP) at 2 months and at the completion of treatment.

Following the World Health Organization (WHO) standard regimen guidelines under Revised National TB Control Program (RNTCP), treatment initiation of newly diagnosed cases was started with four drugs in IP for two months followed by two drugs in continuation phase (CP) for four months (2HRZE/4HR). Retreatment cases were initiated with five drugs in IP (2 months) followed by three drugs in CP for five months (2HRZES/1HRZE/5HRE). Treatment outcomes were defined as per the operational definitions of the program as per WHO guidelines (Table 1) [22].

2.6. Adverse Drug Reaction (ADR) Monitoring. The ADRs were recorded in the suspected adverse drug reporting form, that is, "voluntary reporting of adverse drug reactions by healthcare professionals." Researcher has immediately recorded when any adverse reaction emerges, and routinely 
TABLE 1: Definitions of TB treatment outcome.

\begin{tabular}{ll}
\hline Terms & Definitions \\
Cured & $\begin{array}{l}\text { A PTB patient with bacteriologically confirmed TB at the beginning of treatment who was smear- or } \\
\text { culture-negative in the last month of treatment and on at least one previous occasion }\end{array}$ \\
\hline Treatment completed & $\begin{array}{l}\text { A TB patient who has completed treatment without evidence of failure but with no record to show that sputum } \\
\text { smear or culture results in the last month of treatment and on at least one previous occasion were negative, } \\
\text { either because tests were not done or because results are unavailable }\end{array}$ \\
\hline Treatment failed & A TB patient whose sputum smear or culture is positive at month 5 or later during treatment \\
\hline Died & A TB patient who dies for any reason before starting or during the course of treatment \\
\hline Lost to follow-up & A TB patient who did not start treatment or whose treatment was interrupted for 2 consecutive months or more \\
\hline Not evaluated & $\begin{array}{l}\text { A TB patient for whom no treatment outcome is assigned. This includes cases "transferred out" to another } \\
\text { treatment unit as well as cases for which the treatment outcome is unknown to the reporting unit }\end{array}$ \\
\hline Treatment success & The sum of cured and treatment completed \\
\hline
\end{tabular}

patients were closely supervised until completion of anti-TB medication.

ADR was defined as a response which is noxious, unintended, and occurs at doses normally used in humans [23]. Serious adverse events (SAE) are any untoward medical occurrence that, at any dose, is life-threatening or results in hospitalization or prolongation of existing hospital stay, persistent or significant disability, or death [23]. We considered liver dysfunction after an increase in serum alanine aminotransferase (ALT), or total bilirubin greater than two times the upper limit of normal (ULN), irrespective of the symptoms, in our study [24]. According to American Thoracic Society (ATS) guideline, ALT elevation more than three times the ULN in the presence of hepatitis symptoms and/or jaundice or five times the ULN in the absence of symptoms needs interruption and, generally, a modified regimen is used [25]. Except liver dysfunction, other ADRs including rashes, peripheral neuropathy, joint pain, gastrointestinal disorder, and others were determined based on symptoms/clinical examination as well as medical records.

In case of identification of suspected ADRs, the patients were followed up until resolution or end of TB therapy. Severity of ADRs was symptoms-based, as mild reaction shows no immediate modification of the standard regimen, and moderate reaction may require preventive measures, interruption, dose reduction, drug replacement, and discontinuation of anti-TB drugs [26]. Add-on medication includes antiemetic for relieving minor gastrointestinal reactions (nausea or vomiting) or an antihistaminic agent for reducing minor allergic reactions being manifested as itching. However, generalized erythematous rash, associated with fever and/or mucous membrane, lead to discontinuation of all drugs immediately [27]. Philadelphia tuberculosis control program suggested discontinuation of the drugs if skin reaction appeared till it gets resolved. Further, identification of the causative agents is recommended by rechallenging each drug [28]. Dosage of pyrazinamide and/or ofloxacin should be reduced or the drug withheld temporarily, if arthralgia is not responding to NSAIDs. In renal impairment, the dose of aminoglycosides may be reduced or replaced with other potent nonnephrotoxic antituberculosis drugs. Further, dose adjustment is required with ethambutol, quinolones, cycloserine and PAS in presence of mild to moderate renal impairment [29].

2.7. Statistical Analysis. Data collected were analyzed using Statistical Package for Social Science (SPSS) version 21.0. The finding of patients having TB with DM was compared with those of TB without DM. Categorical variables were compared by chi square test and continuous variables by Student's $t$-test. PTB patients were classified further as sputum positive or negative patients. Odds ratio (OR) was determined for sputum conversion and treatment outcome in DM and non-DM TB patients using logistic regression analysis. Risk factors associated with sputum conversion and treatment outcome were assessed by multiple logistic regression. Patient age, sex, body mass index, TB history, habitual risk, and clinical presentation were included as independent variables in logistic regression models. Further, factors influencing the anti-TB ADRs were analyzed by logistic regression. A twotailed $p$ value of $<0.05$ was considered significant.

\section{Results}

3.1. Patient Characteristics. A total of 546 new and retreatment cases were diagnosed and subsequently managed at all three DOTS centers between the study periods. The patients who fulfill the inclusion criteria were recruited for this study. The flow diagram of subject inclusion is depicted in Figure 1. We recruited 316 patients with PTB and EPTB of both categories I and II during the study period from respective DOTS centers. Among all patients, 191 (60.4\%) were diagnosed with PTB and 125 (39.6) with EPTB. Out of these, $15.8 \%$ (50/316) were diagnosed with DM, of which 9.49\% (30/316) were diagnosed prior to TB diagnosis and the remaining $6.33 \%(20 / 316)$ at the time of DM screening at treatment initiation. The DM was more in PTB patients (19.4\%) as compared to EPTB patients (9.6\%). A comparison of patients with and without DM is depicted in Table 2. Our result shows that DM patients were more likely to be male, be of older age, and have higher mean BMI. The significant proportion of TB appeared to increase with age in DM patients compared to non-DM patients. However, both 


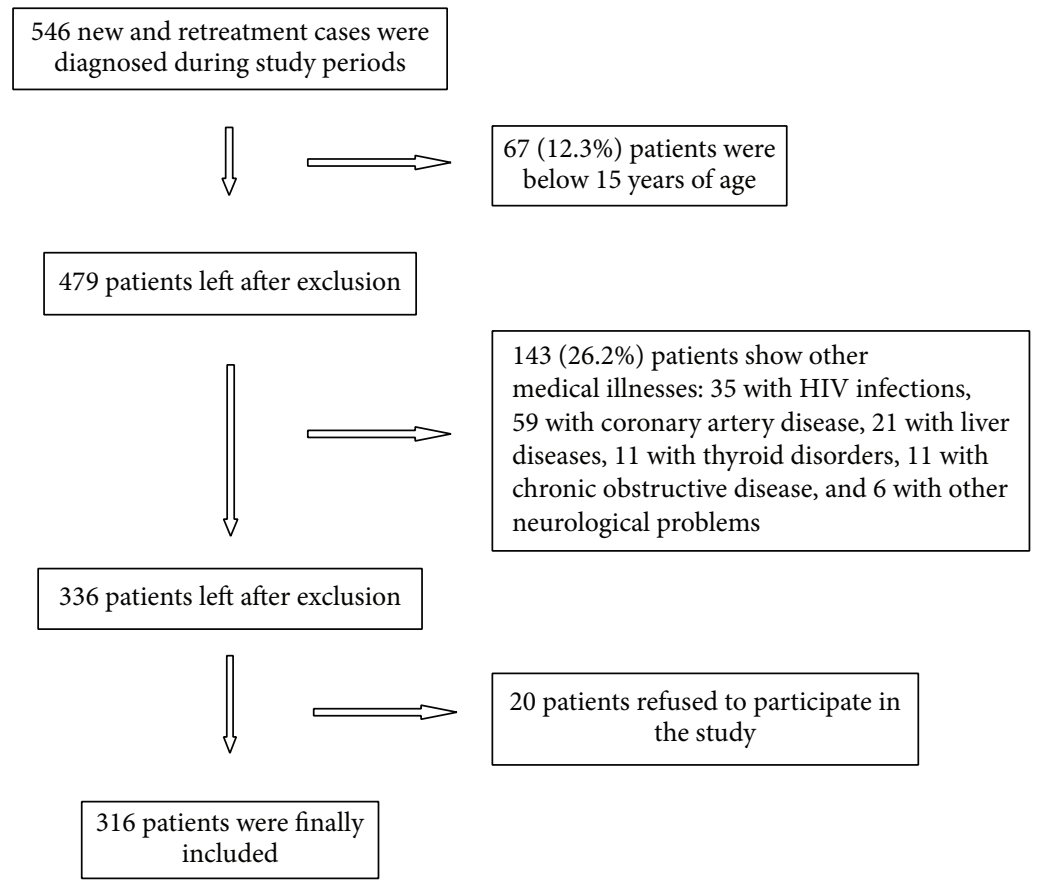

FIGURE 1: Flow diagram of study participants.

groups showed similarities in terms of sex, religion, family history of TB, and type of TB cases (new or retreatment).

Majority of patients in both groups have shown common TB symptoms. Patients with DM presented with more symptoms of dyspnea, chest pain, and hemoptysis while the remaining other symptoms including cough, weight losses, anorexia, and evening fever were predominant in non-DM patients as presented in Table 2. Newly diagnosed DM patients were confirmed to be type $2 \mathrm{DM}$, while among known DM patients, except one all belong to type 2 DM category. Regarding the management of DM with hypoglycemic agents, we found that common medication includes biguanides (40\%), sulphonylureas (24\%), insulin (08\%), thiazolidinedione (02\%), and gliptins (02\%). However few patients $(06 \%)$ were receiving herbal medicine for DM management. Four patients (08\%) underwent only dietary management and 18 (36\%) were advised for exercise also.

\subsection{Treatment Outcomes. Of the total $191 \mathrm{PTB}$ (31 and 6} were smear positive and smear negative in DM group, resp., and 109 and 45 were smear positive and smear negative in non-DM group, resp.) patients, 177 (92.7\%) had completed treatment while 18 (4, loss to follow-up; 10, died; and 4, treatment regimen changed) did not complete it. However all EPTB patients have successfully completed the treatment. $232(73.4 \%)$ patients initiated treatment within 07 days of their diagnosis and all received DOTS regimen. The sputum conversion and treatment outcomes of PTB patients during and at the end of treatment were shown in Table 3 . Higher proportions $(5.6 \%)$ of DM patients were lost to followup during the course of treatment but none experienced treatment regimen change or multidrug resistant TB (MDRTB) compared to non-DM patients (2.7\%) (Table 3). As previously reported, 10 patients died during the treatment of which one $(2.8 \%)$ belongs to DM and the remaining $9(6.0 \%)$ belong to no-DM group. Before completion of IP, one patient died in DM group, while 3 died and 1 was diagnosed with MDR in no-DM group. Microscopic examination of sputum samples at 2 months reveals higher sputum positivity in DM (27.8\%) as compared to no-DM $(24.7 \%)$ patients. Logistic regression analysis showed that DM with TB patients had a higher probability of delayed sputum conversion (OR: 1.247, 95\% CI: 0.539-2.886) and poor treatment outcomes (OR: 1.176, 95\% CI: $0.310-4.457)$ as compared to no-DM patients (Tables 3).

The association of different variables with the treatment outcome has been presented in Table 4. A statistically significant difference was obtained in the TB category, types of TB, and smoking history in the outcome analysis. Baseline clinical characteristic such as fever, dyspnea, and chest pain were also significantly associated with the treatment outcome. In Table 5, all independent variables were examined in multiple logistic regression analysis to find the association of these variables and sputum conversion $>60$ days and poor treatment outcome at the end of treatment.

3.3. ADR Incidence. A total of 224 patients presented with at least one ADR (224/316, i.e., 70.9\%), of which 178 (178/266, i.e., 66.9\%) had no DM and 46 (46/50, i.e., 92.0\%) had DM. The median duration $( \pm S D)$ between onset of anti-TB treatment and first-time adverse reaction occurrence was 14 $( \pm 14.63)$ and $14( \pm 14.06)$ days in DM and no-DM group, respectively. DM patients were commonly encountered with 
TABLE 2: Characteristics of enrolled patients at baseline.

\begin{tabular}{|c|c|c|c|}
\hline Variables & TB with DM $(n=50)$ & TB without DM $(n=266)$ & $p$ value \\
\hline Male & $27(54.0)$ & $148(55.6)$ & \multirow{2}{*}{0.831} \\
\hline Female & $23(46.0)$ & $118(44.4)$ & \\
\hline Age (years) $($ mean $\pm S D)$ & $44.04 \pm 14.04$ & $30.96 \pm 12.89$ & $<0.001^{* * *}$ \\
\hline Family history of TB & $05(10.00)$ & $38(14.28)$ & 0.417 \\
\hline Mean BMI $\left(\mathrm{kg} / \mathrm{m}^{2}\right)($ mean $\pm \mathrm{SD})$ & $19.81 \pm 3.62$ & $17.17 \pm 3.26$ & 0.307 \\
\hline Marital status & & & $<0.001^{* * *}$ \\
\hline Married & $45(90.0)$ & $164(61.6)$ & \\
\hline Unmarried & $02(4.0)$ & $99(37.2)$ & \\
\hline Widow/divorced/other & $03(6.0)$ & $03(1.12)$ & \\
\hline Religion & & & 0.595 \\
\hline Hindu & $41(82.0)$ & $226(84.9)$ & \\
\hline Muslim & $8(16.0)$ & $38(14.3)$ & \\
\hline Christian & $1(2.0)$ & $2(0.75)$ & \\
\hline Type of TB cases & & & 0.799 \\
\hline New & $41(82)$ & $214(80.4)$ & \\
\hline Retreatment & $09(18)$ & $52(19.6)$ & \\
\hline \multicolumn{4}{|l|}{ Habitual risk } \\
\hline Alcohol & $22(44.0)$ & $90(33.8)$ & 0.168 \\
\hline Smoking & $15(30.0)$ & $56(21.0)$ & 0.164 \\
\hline Chewing & $08(16.0)$ & $45(16.9)$ & 0.873 \\
\hline Literacy level & & & $0.003^{* *}$ \\
\hline Illiterate & $17(34.0)$ & $33(12.4)$ & \\
\hline Primary school & $01(2.0)$ & $10(3.8)$ & \\
\hline Middle school & $14(28.0)$ & $66(24.8)$ & \\
\hline High school & $13(26.0)$ & $95(35.7)$ & \\
\hline Intermediate & $04(8.0)$ & $44(16.5)$ & \\
\hline Graduate and professional degree & $01(2.0)$ & $18(6.8)$ & \\
\hline \multicolumn{4}{|l|}{ Sign and symptom } \\
\hline Cough & $36(72.0)$ & $208(78.2)$ & 0.329 \\
\hline Loss of weight (more than $5 \mathrm{~kg}$ ) & $39(78.0)$ & $249(93.6)$ & $0.003^{* *}$ \\
\hline Anorexia & $31(62.0)$ & $180(67.7)$ & 0.721 \\
\hline Evening rise in fever & $28(56.0)$ & $165(62.0)$ & 0.405 \\
\hline Dyspnea & $32(64.0)$ & $137(51.5)$ & 0.530 \\
\hline Chest pain & $28(56.0)$ & $139(52.2)$ & 0.136 \\
\hline Hemoptysis & $21(42.0)$ & $97(36.5)$ & 0.167 \\
\hline
\end{tabular}

TB: tuberculosis; DM: diabetes mellitus; SD: standard deviation; data were analyzed using chi square test between TB with DM and TB without DM groups. $p<0.05$ was considered as significant. ${ }^{* * *} p<0.001 ;{ }^{* *} p<0.01$.

restlessness (42.0\%), peripheral neuropathy (36.0\%), liver disorder (22.0\%), rashes (18.0\%), and other nervous system disorders (40.0\%). Other frequent ADRs that have been experienced were nausea, vomiting, arthralgia, drowsiness, and pain in back and limbs (Table 6). All ADRs were mild to moderate. Rechallenge of suspected drug was not performed in most cases due to safety and practical necessity. Among the collected ADRs, restlessness, hypoglycemia, back pain, and feet pain were significantly associated with TB with DM patients. The occurrence of other ADRs was not significantly different between the two groups as presented in Table 6 . Upon subanalysis of ADRs according to the TB category, we found similar ADRs (restlessness, hypoglycemia, back pain, and feet pain) to be significantly more associated with DM patients in both categories I and II. However, no ADRs were significantly different while comparing DM patients of category I and category II as described in Table 7. Further, this study revealed that 53 patients (16.8\%) appeared with one, 63 (19.9\%) with two, 53 (16.8\%) with three, 33 (10.4\%) with four, 11 (3.5\%) with five, and $10(3.2 \%)$ with more than five ADRs, among 224 cases. The number of ADRs among $\mathrm{DM}$ and no-DM patients is given in Table 8. Our analysis also demonstrated that DM patients were encountered with significantly higher number of ADRs as compared to non$\mathrm{DM}$ patients. The frequency of 5 or more ADR incidences was also significantly higher in DM patients $(p \leq 0.001)$. 
TABLE 3: Sputum conversion and treatment outcome of PTB patients with and without DM.

\begin{tabular}{|c|c|c|c|c|}
\hline Variables & $\begin{array}{c}\text { TB with DM }(n=50) \\
n(\%)\end{array}$ & $\begin{array}{c}\text { TB without DM }(n=266) \\
n(\%)\end{array}$ & OR $(95 \% \mathrm{CI})^{\$}$ & $p$ value \\
\hline $\mathrm{PTB}^{\#}$ & $37(74.0)$ & $154(57.9)$ & $2.168(1.102-4.265)$ & \multirow{2}{*}{$0.023^{!}$} \\
\hline $\mathrm{EPTB}^{\&}$ & $13(26.0)$ & $112(42.1)$ & 1 & \\
\hline \multicolumn{5}{|c|}{$\begin{array}{l}\text { PTB patients ( } n=36 \text { in TB with DM group } \\
\text { and } n=150 \text { in TB without DM group) }{ }^{*}\end{array}$} \\
\hline \multicolumn{5}{|l|}{ Sputum status at 2 months } \\
\hline Sputum positive & $10(27.8)$ & $37(24.7)$ & $1.222(0.537-2.779)$ & \multirow{2}{*}{0.633} \\
\hline Sputum conversion & $26(72.2)$ & $113(75.3)$ & 1 & \\
\hline Treatment outcomes & & & & 0.428 \\
\hline Successful outcomes & & & 1 & \\
\hline Cured & $26(72.2)$ & $93(62.0)$ & & \\
\hline Treatment completed & $06(16.7)$ & $43(28.7)$ & & \\
\hline Poor outcomes & & & $1.176(0.310-4.457)$ & \\
\hline Default & $02(5.6)$ & $02(1.3)$ & & \\
\hline Failure & $02(5.6)$ & $03(2.0)$ & & \\
\hline Died & $01(2.8)$ & $09(6.0)$ & & \\
\hline Shifted to MDR & 00 & $04(2.7)$ & & \\
\hline
\end{tabular}

We have further found that $4.43 \%(14 / 316)$ of total patients required modification in their anti-TB treatment due to ADRs. Most of these modifications were in the form of add-on therapy, where antihistamines and antiemetic were prescribed by the clinician. Few were also prescribed with pyridoxine to avoid the peripheral neuropathy. Occurrence of side effect was associated with being male (OR, $2.01395 \%$ CI: 0.906-4.473), being in category I (OR, 2.165 95\% CI: 1.004-4.670), having PTB (OR, 1.071 95\% CI: 0.555-2.065), being married (OR 1.618 95\%: 0.804-3.258), being Hindu (OR 1.131 95\% CI: 0.498-2.567), and having DM (OR 3.578 95\% CI: 1.114-11.494) in multivariate analysis. Except category I $(p=0.049)$ and DM $(p=0.032)$, no other variables were significantly associated with ADR incidence as presented in Table 9. The odds of developing ADRs were 3.5 (OR; 3.574; 95\% C.I; (1.114-11.494)) times higher in DM than no-DM patients.

\section{Discussion}

Despite the evidences of concurrent increase in the incidence of TB and DM cases, there is very limited data available from north Indian population presenting the association of this comorbidity. The Union/World diabetes foundation has acknowledged the need of more epidemiological research to determine the TB burden attributed to DM, particularly in developing countries. The present work highlights the consequences of DM on the disease presentation, treatment outcome, and ADRs of anti-TB medication.
The overall prevalence of DM in our study was found to be $15.8 \%$ which is well above the global DM prevalence $(9.0 \%)$ among general population [30]. Similar DM occurrence among TB patients has also been demonstrated in other tropical countries [31, 32]. In previous literatures, a wide range of DM prevalence from $1.9 \%$ to $35 \%$ has been reported among TB patients [1]. Further, we have recorded $40 \%$ newly diagnosed DM patients; probably they remain unrecognized due to delay in DM screening [33]. Few studies from Tanzania and Indonesia have reported $73 \%$ and $61 \%$ of newly diabetics diagnosed concurrent with active TB, respectively $[10,34]$. This again confers the need of expanded medical attention in relation to DM screening and its management for improvement of TB treatment outcome.

The data in this study showed that PTB patients with DM have reduced rate of sputum conversion with higher probability of poor treatment outcome, namely, default, death, failure, and shifting to MDR category, than patients without DM. Consistent with the previous studies, we have also found more severe clinical manifestation among patients with TB and DM $[15,35]$. The finding regarding sputum conversion at the end of 60 days also agrees with other studies [17]. The independent risk of poor outcome among PTB patients associated with DM in our study was $1.176(95 \%$ CI: 0.310-4.457), which is little lower than the previously reported pooled risk of 1.69 (95\% CI: 1.36-2.12) associated with the TB treatment failure and death [17]. This difference may be attributed to relatively smaller sample size in our study. 
TABLE 4: Association of treatment outcomes and different variables in TB patients.

\begin{tabular}{|c|c|c|c|}
\hline Variables & $\begin{array}{l}\text { Poor outcome } \\
\quad(n=23)\end{array}$ & $\begin{array}{l}\text { Treatment success } \\
\quad(n=293)\end{array}$ & $p$ value \\
\hline Sex & & & 0.32 \\
\hline Male & $15(65.2)$ & $160(54.6)$ & \\
\hline Female & $08(34.8)$ & $133(45.4)$ & \\
\hline Category & & & $<0.001^{* * *}$ \\
\hline Category I & $12(52.2)$ & $243(82.9)$ & \\
\hline Category II & $11(47.8)$ & $50(17.1)$ & \\
\hline TB types & & & $<0.001^{* * *}$ \\
\hline PTB & $23(100)$ & $168(57.3)$ & \\
\hline ЕРТВ & 00 & $125(42.7)$ & \\
\hline Family history of TB & & & 0.94 \\
\hline Yes & $03(13.1)$ & $40(13.6)$ & \\
\hline No & $20(86.9)$ & $253(86.3)$ & \\
\hline Alcoholic history & & & 0.08 \\
\hline Yes & $12(52.2)$ & $100(34.1)$ & \\
\hline No & $11(47.8)$ & $193(65.9)$ & \\
\hline Smoking history & & & $0.04^{*}$ \\
\hline Yes & 09 (39.1) & $62(21.2)$ & \\
\hline No & $14(60.8)$ & $231(78.8)$ & \\
\hline Chewing history & & & 0.51 \\
\hline Yes & $05(21.7)$ & $48(16.4)$ & \\
\hline No & $18(78.2)$ & $245(83.6)$ & \\
\hline$\overline{\mathrm{ADR}}$ & & & 0.74 \\
\hline Present & $17(73.9)$ & $207(70.6)$ & \\
\hline Absent & $06(26.1)$ & $86(29.3)$ & \\
\hline Cough & & & 0.25 \\
\hline Present & $20(86.9)$ & $224(76.4)$ & \\
\hline Absent & $03(13.1)$ & $69(23.5)$ & \\
\hline Weight loss & & & $0.02^{*}$ \\
\hline Present & $18(78.3)$ & $270(92.1)$ & \\
\hline Absent & $05(21.7)$ & $23(7.8)$ & \\
\hline Anorexia & & & 0.09 \\
\hline Present & $19(82.6)$ & $192(65.5)$ & \\
\hline Absent & $04(17.4)$ & $101(34.5)$ & \\
\hline Fever & & & $0.008^{* *}$ \\
\hline Present & $20(86.9)$ & $173(59.1)$ & \\
\hline Absent & $03(13.0)$ & $120(40.9)$ & \\
\hline Dyspnea & & & $0.01^{*}$ \\
\hline Present & $18(78.3)$ & $151(51.5)$ & \\
\hline Absent & $05(21.7)$ & $142(48.4)$ & \\
\hline Chest pain & & & $0.04^{*}$ \\
\hline Present & $17(73.9)$ & $150(51.2)$ & \\
\hline Absent & $06(26.1)$ & $143(48.8)$ & \\
\hline Hemoptysis & & & 0.13 \\
\hline Present & $12(52.2)$ & $106(36.2)$ & \\
\hline Absent & $11(47.8)$ & $187(63.8)$ & \\
\hline $\mathrm{DM}$ & & & 0.42 \\
\hline Present & 05 (21.7) & $45(15.4)$ & \\
\hline Absent & $18(78.3)$ & $248(84.6)$ & \\
\hline
\end{tabular}

Poor outcome: default, death, failure, and regimen changed; treatment success: cured and treatment completed; PTB: pulmonary tuberculosis; EPTB: extrapulmonary tuberculosis; DM: diabetes mellitus; ADR: adverse drug reaction; data was analyzed using chi square test. $p \leq 0.05$ was considered as significant. ${ }^{* * *} p<0.001,{ }^{* *} p<0.01$, and ${ }^{*} p<0.05$.

Analyzing symptoms associated with TB, compared to non-DM patients, weight loss was more frequent in $\mathrm{TB}$
TABle 5: Association of clinical manifestations with sputum positivity and treatment outcomes among TB patients by multivariate analyses.

\begin{tabular}{lcc}
\hline Variables & $\begin{array}{c}\text { Sputum positive }>60 \text { days } \\
\text { OR }(95 \% \mathrm{CI})\end{array}$ & $\begin{array}{c}\text { Poor outcome } \\
\text { OR }(95 \% \mathrm{CI})\end{array}$ \\
\hline DM & $0.633(0.206-1.949)$ & $0.714(0.155-3.279)$ \\
Men & $1.284(0.327-4.430)$ & $0.312(0.055-1.762)$ \\
Age & $0.992(0.957-1.029)$ & $0.960(0.904-1.020)$ \\
Category & $1.685(0.647-4.391)$ & $0.838(0.243-2.888)$ \\
BMI & $0.914(0.794-1.052)$ & $1.185(0.970-1.447)$ \\
TB history & $1.797(0.491-6.582)$ & $2.591(0.260-25.821)^{*}$ \\
Alcohol intake & $0.880(0.280-2.764)$ & $0.674(0.177-2.558)$ \\
Smoking & $1.811(0.673-4.877)$ & $0.752(0.236-2.390)$ \\
Chewing & $0.689(0.252-1.883)$ & $0.778(0.241-2.543)$ \\
ADR incidence & $1.797(0.491-6.582)^{*}$ & $0.642(0.187-2.207)$ \\
Weight gain & $0.914(0.141-5.915)$ & $0.708(0.059-8.521)$ \\
Anorexia & $1.165(0.060-22.585)$ & $0.558(0.048-3.124)$ \\
Fever & $2.176(0.321-14.734)^{*}$ & $0.814(0.093-2.155)$ \\
Dyspnea & $0.138(0.010-1.902)$ & $1.973(0.337-5.871)^{*}$ \\
Chest pain & $0.090(0.010-0.847)$ & $1.370(0.485-6.143)$ \\
Hemoptysis & $1.582(0.062-0.551)$ & $0.813(0.291-2.275)$ \\
\hline
\end{tabular}

OR: odds ratio; CI: confidence interval; BMI: body mass index; ADR: adverse drug reaction; DM: diabetes mellitus. All independent variables were analyzed using multiple logistic regression analysis to calculate the odds ratio. The OR presented is adjusted for age, gender, and BMI in logistic regression analysis. ${ }^{*} p \leq 0.05$ was considered as significant.

patients with DM. Though weight loss is associated with both TB and DM, we have found relatively lesser weight loss in DM patients in our study. Contrary to this, Alisjahbana et al. (2010) have reported more weight loss in DM patients [10]. Few authors have revealed that clinical characteristics of TB do not differ among DM and non-DM patients [33, 35]. In agreement with others, we have also found less extrapulmonary involvement among DM than in no DM patients [33]. Contradictory reports are found with reference to the rate of positive smears at the time of diagnosis to different populations. We have observed higher positive smears among DM patients $(22.6 \%)$ as compared to negative smears. Alisjahbana et al. (2007) reported a higher frequency of negative sputum smears among DM patients [10], while few showed no association between DM and sputum status of patients [36].

Current literatures on the effect of DM on sputum conversion are also conflicting. The independent risk of sputum positivity, associated with DM, is 1.176 (95\% CI: 0.310-4.457) at the end of 60 days in our study. Few studies did not reveal any relation between DM and sputum conversion rate at the end of 60 days $[1,14]$. Probably the sputum positivity at the end of IP is more likely to be associated with poorly controlled DM status.

The effectiveness of DOTS therapy has been well established worldwide; however, a combination regimen is often a concern to evaluate the safety of a given drug. Pharmacokinetic interactions along with thorough knowledge of possible 
TABLE 6: Total ADRs collected from all patients.

\begin{tabular}{|c|c|c|c|c|c|}
\hline \multirow[b]{2}{*}{$\mathrm{ADR}$} & \multicolumn{2}{|c|}{ TB with DM $(n=50)$} & \multicolumn{2}{|c|}{ TB without DM $(n=266)$} & \multirow[b]{2}{*}{$p$ value } \\
\hline & $\begin{array}{c}\text { ADR incidence } \\
n(\%)\end{array}$ & $\begin{array}{l}\text { Onset time, days } \\
\text { (median, IQR) }\end{array}$ & $\begin{array}{c}\text { ADR incidence } \\
n(\%)\end{array}$ & $\begin{array}{l}\text { Onset time, days } \\
\text { (median, IQR) }\end{array}$ & \\
\hline Nausea and vomiting & $13(26.0)$ & $12(7-26)$ & $50(18.80)$ & $14(5-32)$ & 0.242 \\
\hline Rashes & $12(24.0)$ & $16(10-29)$ & $62(23.31)$ & $13(5-45)$ & 0.916 \\
\hline Peripheral neuropathy & $18(36.0)$ & $17(7-50)$ & $62(23.31)$ & $15(10-39)$ & 0.058 \\
\hline Liver injury & $11(22.0)$ & $25(17-54)$ & $48(18.04)$ & $32(25-65)$ & 0.510 \\
\hline Restlessness & $21(42.0)$ & $10(5-25)$ & $48(18.04)$ & $08(5-21)$ & $<0.001^{* * *}$ \\
\hline GI problem $\%$ & $07(14.0)$ & $12(5-40)$ & $23(8.65)$ & $16(11-32)$ & 0.236 \\
\hline Hypoglycemia & $02(4.0)$ & $06(3-10)$ & 00 & 00 & $0.001^{* * *}$ \\
\hline Joint pain ${ }^{\#}$ & $07(14.0)$ & $20(11-45)$ & $32(12.03)$ & $14(10-55)$ & 0.698 \\
\hline Drowsiness & $05(10.0)$ & $11(5-27)$ & $41(15.41)$ & $08(3-21)$ & 0.319 \\
\hline Back pain & $07(14.0)$ & $37(15-55)$ & 09 (3.38) & $22(12-51)$ & $0.002^{* *}$ \\
\hline Feet pain & $05(10.0)$ & $30(25-39)$ & $06(2.25)$ & $21(18-45)$ & $0.006^{*}$ \\
\hline Body ache & $05(10.0)$ & $10(7-18)$ & $11(3.48)$ & $18(10-24)$ & 0.083 \\
\hline Blurring vision & $01(2.0)$ & 60 & $03(1.12)$ & $55(42-64)$ & 0.613 \\
\hline Other nervous system disorders $\$$ & $20(40.0)$ & $08(03-18)$ & $74(23.4)$ & $06(15-50)$ & 0.084 \\
\hline
\end{tabular}

TABLE 7: ADR incidence in different categories of TB patients with and without DM.

\begin{tabular}{|c|c|c|c|c|c|c|c|}
\hline \multirow[b]{2}{*}{ ADRs } & \multicolumn{3}{|c|}{ Category I $(n=255)$} & \multicolumn{3}{|c|}{ Category II $(n=61)$} & \multirow[b]{2}{*}{$p$ value $^{3}$} \\
\hline & $\begin{array}{c}\text { TB with DM } \\
(n=41) \\
\text { ADR incidence, } \\
n(\%)\end{array}$ & $\begin{array}{c}\text { TB without DM } \\
(n=214) \\
\text { ADR incidence, } \\
n(\%)\end{array}$ & $p$ value $^{1}$ & $\begin{array}{c}\text { TB with DM } \\
(n=09) \\
\text { ADR incidence, } \\
n(\%)\end{array}$ & $\begin{array}{c}\text { TB without DM } \\
(n=52) \\
\text { ADR incidence, } \\
n(\%)\end{array}$ & $p$ value $^{2}$ & \\
\hline Nausea and vomiting & $11(26.8)$ & $38(17.7)$ & 0.177 & $02(22.2)$ & $12(23.07)$ & 0.955 & 0.775 \\
\hline Rashes & $10(24.4)$ & $43(20.1)$ & 0.534 & $02(22.2)$ & $19(36.5)$ & 0.404 & 0.890 \\
\hline $\begin{array}{l}\text { Peripheral } \\
\text { neuropathy }\end{array}$ & $14(34.1)$ & $45(21.0)$ & 0.068 & $04(44.4)$ & $17(32.7)$ & 0.493 & 0.560 \\
\hline Liver injury & 07 (17.1) & $32(15.0)$ & 0.730 & $04(44.4)$ & $16(30.8)$ & 0.420 & 0.073 \\
\hline Restlessness & $15(36.6)$ & $36(16.8)$ & $0.004^{*}$ & $06(66.6)$ & $12(23.1)$ & $0.008^{*}$ & 0.098 \\
\hline GI problem & $05(12.2)$ & $18(8.4)$ & 0.438 & $02(22.2)$ & $05(9.6)$ & 0.273 & 0.432 \\
\hline Hypoglycemia & $01(2.4)$ & 00 & $0.022^{*}$ & 01 (11.1) & 00 & $0.015^{*}$ & 0.229 \\
\hline Joint pain & $05(12.2)$ & $19(8.9)$ & 0.505 & $02(22.2)$ & $13(25.0)$ & 0.858 & 0.432 \\
\hline Drowsiness & $05(12.2)$ & $31(14.5)$ & 0.700 & 00 & $10(19.2)$ & 0.150 & 0.269 \\
\hline Back pain & $05(12.2)$ & $07(3.3)$ & $0.013^{*}$ & $02(22.2)$ & $02(3.9)$ & $0.040^{*}$ & 0.432 \\
\hline Feet pain & $04(9.7)$ & $06(2.8)$ & $0.036^{*}$ & $01(11.1)$ & 00 & $0.015^{*}$ & 0.902 \\
\hline Body ache & $04(9.7)$ & $07(3.3)$ & 0.061 & $01(11.1)$ & $04(7.7)$ & 0.730 & 0.902 \\
\hline Blurring vision & $01(2.4)$ & $03(1.4)$ & 0.624 & 00 & 00 & 00 & 0.636 \\
\hline $\begin{array}{l}\text { Other nervous system } \\
\text { disorders }\end{array}$ & $16(39.0)$ & $52(24.3)$ & 0.051 & $04(44.4)$ & $22(42.3)$ & 0.905 & 0.764 \\
\hline
\end{tabular}

${ }^{1}$ Data compared between TB with DM and TB without DM group in category I patients.

${ }^{2}$ Data compared between TB with DM and TB without DM group in category II patients.

${ }^{3}$ Data compared between TB with DM patients between category I and category II patients.

ADR: adverse drug reaction; TB: tuberculosis; DM: diabetes mellitus; all data has been analyzed by chi square test; ${ }^{*} p \leq 0.05$ was considered as significant. 
TABLE 8: Number of adverse reactions recorded in DM and non-DM TB patients.

\begin{tabular}{lccc}
\hline $\begin{array}{l}\text { Number of ADRs } \\
\text { reported }\end{array}$ & $\begin{array}{c}\text { TB with DM } \\
n=50\end{array}$ & $\begin{array}{c}\text { TB without DM } \\
n=266\end{array}$ & $p$ value \\
\hline No ADRs & $04(8.0)$ & $89(33.46)$ & $<0.001^{* * *}$ \\
One & $06(12.0)$ & $47(17.67)$ & 0.325 \\
Two & $08(16.0)$ & $55(20.67)$ & 0.448 \\
Three & $13(26.0)$ & $40(15.04)$ & 0.057 \\
Four & $05(10.0)$ & $28(10.53)$ & 0.911 \\
Five & $07(14.0)$ & $04(1.50)$ & $<0.001^{* * *}$ \\
More than five & $07(14.0)$ & $03(1.13)$ & $<0.001^{* * *}$ \\
\hline
\end{tabular}

ADR: adverse drug reaction; TB: tuberculosis; DM: diabetes mellitus; values in parenthesis are expressed in percentage; values were compared by using chi square test. $p \leq 0.05$ was considered as significant. Statistical calculation was performed by using chi square test between the two groups. ${ }^{* * *} p<0.001$.

TABLE 9: Multivariate analysis showing factors associated with TB treatment adverse effect.

\begin{tabular}{lcc}
\hline Variables & OR $(95 \% \mathrm{CI})$ & $p$ value \\
\hline Age & $0.970(0.940-1.001)$ & 0.062 \\
Male & $2.013(0.906-4.473)$ & 0.086 \\
Category I cases & $2.165(1.004-4.670)$ & $0.049^{*}$ \\
PTB & $1.071(0.555-2.065)$ & 0.838 \\
Married & $1.618(0.804-3.258)$ & 0.178 \\
Hindu & $1.131(0.498-2.567)$ & 0.769 \\
BMI & $0.965(0.874-1.065)$ & 0.478 \\
Joint family & $0.725(0.359-1.462)$ & 0.369 \\
Alcoholic history & $0.942(0.427-2.078)$ & 0.883 \\
Smoking history & $0.853(0.411-1.768)$ & 0.669 \\
Chewing history & $0.617(0.275-1.385)$ & 0.242 \\
Family TB history & $0.451(0.184-1.105)$ & 0.081 \\
Presence of DM & $3.578(1.114-11.494)$ & $0.032^{*}$ \\
Cough & $1.104(0.426-2.864)$ & 0.839 \\
Weight loss & $1.326(0.411-4.276)$ & 0.637 \\
Anorexia & $0.309(0.059-1.607)$ & 0.163 \\
Fever & $1.276(0.383-4.245)$ & 0.691 \\
Dyspnea & $0.733(0.278-1.934)$ & 0.531 \\
Chest pain & $0.857(0.279-2.633)$ & 0.788 \\
Hemoptysis & $1.069(0.564-2.026)$ & 0.839 \\
\hline OR: &
\end{tabular}

OR: odds ratio; TB: tuberculosis; DM: diabetes mellitus.

All independent variables were analyzed using multiple logistic regression analysis. ${ }^{*} p<0.05$ was considered as significant. The OR presented is adjusted for age, gender, and BMI in logistic regression analysis.

side effects will always enable a clinician to treat patients with anti-TB drugs more safely. The overall ADR in total patients was $70.9 \%$ including $92.0 \%$ and $69.9 \%$ in DM and nonDM patients, respectively. A few previous studies analyzed adverse events during anti-TB treatment in India, but the subjects were recruited from single location and sample sizes were small. Moreover, to date there are no published reports on incidence of adverse events during anti-TB treatment in DM and no-DM patients. The ADR incidences observed in this study are similar to previously published studies in Bangladesh, Nepal, and India [37-39]. Gholami et al. (2006) revealed 54.3\% ADR incidences, associated with TB medications, in Iranian patients [40]. Presence of DM is significantly associated (OR: 3.578 95\% CI: 1.114-11.494, $p=$ 0.032) with anti-TB ADR, which may be attributed to the concomitant antidiabetic medications. However, we could not perform the subanalyses of DM pharmacotherapy in patients compared to no-DM patients. Lower plasma level of rifampicin has been reported in DM patients; however, the exact mechanism is yet to be elucidated [41, 42]. To our knowledge, no data exists concerning the incidence of ADRs in DOTS patients, particularly in those with DM. It would be mandatory to ascertain individuals having more risk of developing ADRs after initiating anti-TB treatment and they should be followed up by closer monitoring.

4.1. Strength and Limitations. These findings provided significant evidence and contributed to a better understanding and proper management in the course of TB among DM patients. Instead of medical records, we relied on laboratory tests to determine DM status. Diagnosis of DM was based on repeated glucose measurements rather than one-point estimation to avoid the misclassification of cases of DM due to a mixture of biological variation in blood glucose levels and measurement error. We referred newly diagnosed DM patients to the healthcare physician for DM management and also recorded the DM pharmacotherapy in this study. The side effects related to anti-TB drugs are inclusive of the entire treatment duration rather than those evolved in the initial phase of anti-TB therapy.

The results should be interpreted in the light of few limitations as the findings of this study are clearly not representative of all tuberculosis patients. We restricted our study to new and retreatment TB cases (category I and category II) only leaving other classes of TB patients. We did not include MDR TB patients; hence we lack the data on susceptibility to antiTB drugs in TB patients complicated with DM. Further, the radiological interpretations were not obtained from most of the patients. Smear cultures were not collected as they are not performed routinely in TB clinics and the treatment outcome was mainly based on the sputum smear results. We could not assess the relationship between impaired glycaemia and diabetes and pulmonary TB. Information on severity of DM and their association with TB treatment outcome could not be evaluated in this study. This work was mainly confined in urban including slum area of south Delhi. Further prospective longitudinal interventional randomized studies covering larger sample including urban and rural populations of different subject group are necessarily recommended.

\section{Conclusion}

Active screening measures for DM are recommended in patients with TB which could improve the diagnosis and early management of DM complications. Treatment outcomes in patients with DM presence have been a subject of debate. Moreover, there is insufficient number of studies available in settings with high burdens of both diseases. There is further need of studying the effect of long-term evolution of 
DM control and associated complications on TB treatment outcome. Glycemic control should be strictly maintained, particularly, during the initial intensive phase for better outcome in patients with DM.

\section{Competing Interests}

All authors declare that they have no competing interests.

\section{Acknowledgments}

The authors are thankful to the study participants, NITRD, for providing research infrastructures. They are also thankful to Sun Pharma, India, for providing assistantship for this project under the joint collaboration for Ph.D. program with Hamdard University, India.

\section{References}

[1] K. E. Dooley and R. E. Chaisson, "Tuberculosis and diabetes mellitus: convergence of two epidemics," The Lancet Infectious Diseases, vol. 9, no. 12, pp. 737-746, 2009.

[2] Z. A. Bukhary, "Rediscovering the association between tuberculosis and diabetes mellitus: a perspective," Journal of Taibah University Medical Sciences, vol. 3, no. 1, pp. 1-6, 2008.

[3] P. Baghaei, M. Marjani, P. Javanmard, P. Tabarsi, and M. R. Masjedi, "Diabetes mellitus and tuberculosis facts and controversies," Journal of Diabetes and Metabolic Disorders, vol. 12, no. 1, article 58, 2013.

[4] International Diabetes Federation, IDF Diabetes Atlas, International Diabetes Federation, Brussels, Belgium, 6th edition, 2015.

[5] WHO, Tuberculosis Control in the South-East Asia Region. Annual TB Report, WHO, Geneva, Switzerland, 2015.

[6] C. Y. Jeon and M. B. Murray, "Diabetes mellitus increases the risk of active tuberculosis: a systematic review of 13 observational studies," PLoS Medicine, vol. 5, no. 7, article e152, 2008.

[7] The Lancet Diabetes \& Endocrinology, "Diabetes and tuberculosis-a wake-up call," The Lancet Diabetes \& Endocrinology, vol. 2, no. 9, p. 677, 2014.

[8] S. Balakrishnan, S. Vijayan, S. Nair et al., "High diabetes prevalence among tuberculosis cases in Kerala, India," PLoS ONE, vol. 7, no. 10, Article ID e46502, 2012.

[9] V. Viswanathan, S. Kumpatla, V. Aravindalochanan et al., "Prevalence of diabetes and pre-diabetes and associated risk factors among tuberculosis patients in India," PLOS ONE, vol. 7, no. 7, Article ID e41367, 2012.

[10] B. Alisjahbana, E. Sahiratmadja, E. J. Nelwan et al., "The effect of type 2 diabetes mellitus on the presentation and treatment response of pulmonary tuberculosis," Clinical Infectious Diseases, vol. 45, no. 4, pp. 428-435, 2007.

[11] N. K V, K. Duraisamy, S. Balakrishnan et al., "Outcome of tuberculosis treatment in patients with diabetes mellitus treated in the revised national tuberculosis control programme in Malappuram District, Kerala, India," PLoS ONE, vol. 8, no. 10, Article ID e76275, 2013.

[12] M. E. Jiménez-Corona, L. P. Cruz-Hervert, L. García-García et al., "Association of diabetes and tuberculosis: impact on treatment and post-treatment outcomes," Thorax, vol. 68, no. 3 , pp. 214-220, 2013.

[13] A. M. Morsy, H. H. Zaher, M. H. Hassan, and A. Shouman, "Predictors of treatment failure among tuberculosis patients under DOTS strategy in Egypt," Eastern Mediterranean Health Journal, vol. 9, no. 4, pp. 689-701, 2003.

[14] K. E. Dooley, T. Tang, J. E. Golub, S. E. Dorman, and W. Cronin, "Impact of diabetes mellitus on treatment outcomes of patients with active tuberculosis," The American Journal of Tropical Medicine and Hygiene, vol. 80, no. 4, pp. 634-639, 2009.

[15] C. S. Wang, C. J. Yang, H. C. Chen et al., "Impact of type 2 diabetes on manifestations and treatment outcome of pulmonary tuberculosis," Epidemiology and Infection, vol. 137, no. 2, pp. 203-210, 2009.

[16] Y.-Y.Xia and S.-Y. Zhan, "Systematic review of anti-tuberculosis drug induced adverse reactions in China," Zhonghua Jie He He Hu Xi Za Zhi, vol. 30, no. 6, pp. 419-423, 2007.

[17] M. A. Baker, A. D. Harries, C. Y. Jeon et al., "The impact of diabetes on tuberculosis treatment outcomes: a systematic review," BMC Medicine, vol. 9, article 81, 2011.

[18] B. I. Restrepo, S. P. Fisher-Hoch, J. G. Crespo et al., “Type 2 diabetes and tuberculosis in a dynamic bi-national border population," Epidemiology \& Infection, vol. 135, no. 3, pp. 483491, 2007.

[19] American Diabetes Association, "Standards of medical care in diabetes-2016: summary of revisions," Diabetes Care, vol. 39, supplement 1, pp. S4-S5, 2016.

[20] WHO, Definition and Diagnosis of Diabetes Mellitus and Intermediate Hyperglycaemia: Report of a WHO, WHO, Geneva, Switzerland, 2006.

[21] World health Organization (WHO), Laboratory Services in Tuberculosis Control Microscopy Part II, World health Organization, Geneva, Switzerland, 1998.

[22] RNTCP, Revised National Tuberculosis Control Programme, Training Course for Programme Manager (Modules 1-4), 2011.

[23] I. R. Edwards and J. K. Aronson, "Adverse drug reactions: definitions, diagnosis, and management," The Lancet, vol. 356, no. 9237, pp. 1255-1259, 2000.

[24] X. Lv, S. Tang, Y. Xia et al., "Adverse reactions due to directly observed treatment strategy therapy in Chinese tuberculosis patients: a prospective study," PLoS ONE, vol. 8, no. 6, Article ID e65037, 2013.

[25] J. J. Saukkonen, D. L. Cohn, R. M. Jasmer et al., "An official ATS statement: hepatotoxicity of antituberculosis therapy," American Journal of Respiratory and Critical Care Medicine, vol. 174, no. 8, pp. 935-952, 2006.

[26] WHO, Treatment of Tuberculosis Guidelines for National Program, WHO/CDS/TB/, Geneva, Switzerland, 3rd edition, 2003.

[27] H. M. Blumberg, W. J. Burman, R. E. Chaisson et al., "American thoracic society/centers for disease control and prevention/infectious diseases society of america: treatment of tuberculosis," American Journal of Respiratory and Critical Care Medicine, vol. 167, no. 4, pp. 603-662, 2003.

[28] PTC. P, "Guidelines for the management of adverse drug effects of antimycobacterial agents," in Program PTC, Lawrence Flick Memorial Tuberculosis Clinic, Philadelphia, Pa, USA, 1998.

[29] RNTCP: DOTS-Plus Guidelines, Central TB Division, Directorate General of Health Services, Ministry of Health \& Family Welfare, Nirman Bhavan, New Delhi, India, 2010.

[30] World Health Organization (WHO), Global Status Report on Noncommunicable Diseases, World Health Organization, Geneva, Switzerland, 2014.

[31] B. Alisjahbana, R. van Crevel, E. Sahiratmadja et al., "Diabetes mellitus is strongly associated with tuberculosis in Indonesia," International Journal of Tuberculosis and Lung Disease, vol. 10, no. 6, pp. 696-700, 2006. 
[32] D. Duangrithi, V. Thanachartwet, V. Desakorn et al., "Impact of diabetes mellitus on clinical parameters and treatment outcomes of newly diagnosed pulmonary tuberculosis patients in Thailand," International Journal of Clinical Practice, vol. 67, no. 11, pp. 1199-1209, 2013.

[33] R. Ruslami, R. E. Aarnoutse, B. Alisjahbana, A. J. A. M. van der Ven, and R. van Crevel, "Implications of the global increase of diabetes for tuberculosis control and patient care," Tropical Medicine and International Health, vol. 15, no. 11, pp. 1289-1299, 2010.

[34] F. Mugusi, A. B. M. Swai, K. G. M. M. Alberti, and D. G. McLarty, "Increased prevalence of diabetes mellitus in patients with pulmonary tuberculosis in Tanzania," Tubercle, vol. 71, no. 4, pp. 271-276, 1990.

[35] D. Tatar, G. Senol, S. Alptekin, C. Karakurum, M. Aydin, and I. Coskunol, "Tuberculosis in diabetics: features in an endemic area," Japanese Journal of Infectious Diseases, vol. 62, no. 6, pp. 423-427, 2009.

[36] F. Bacakoğlu, Ö. K. Başoğlu, G. Çok, A. Sayiner, and M. Ateş, "Pulmonary tuberculosis in patients with diabetes mellitus," Respiration, vol. 68, no. 6, pp. 595-600, 2001.

[37] Z. A. Begum, S. Sultana, B. U. Umar, A. H. Ferdous, M. K. Uddin, and S. M. Hasibul Islam, "Study of adverse drug reactions in out-patient departments of a teaching hospital," Bangladesh Journal of Pharmacology, vol. 7, no. 2, pp. 104-107, 2012.

[38] D. Koju, B. Rao, B. Shrestha, R. Shakya, and R. Makaju, “Occurrence of side effects from anti-tuberculosis drugs in urban Nepalese population under DOTS treatment," Kathmandu University Journal of Science, Engineering and Technology, vol. 1, pp. $1-2,2005$.

[39] M. Sharma, K. U. Khayyam, V. Kumar, F. Imam, K. Pillai, and D. Behera, "Influence of honey on adverse reactions due to anti-tuberculosis drugs in pulmonary tuberculosis patients," Continental Journal of Pharmacology and Toxicology Research, vol. 2, pp. 6-11, 2008.

[40] K. Gholami, E. Kamali, M. Hajiabdolbaghi, and G. Shalviri, "Evaluation of anti-tuberculosis induced adverse reactions in hospitalized patients," Pharmacy Practice, vol. 4, no. 3, pp. 134138, 2006.

[41] S. K. Heysell, J. L. Moore, S. J. Keller, and E. R. Houpt, "Therapeutic drug monitoring for slow response to tuberculosis treatment in a state control program, Virginia, USA," Emerging Infectious Diseases, vol. 16, no. 10, pp. 1546-1553, 2010.

[42] P. R. Gwilt, R. R. Nahhas, and W. G. Tracewell, "The effects of diabetes mellitus on pharmacokinetics and pharmacodynamics in humans," Clinical Pharmacokinetics, vol. 20, no. 6, pp. 477490, 1991. 

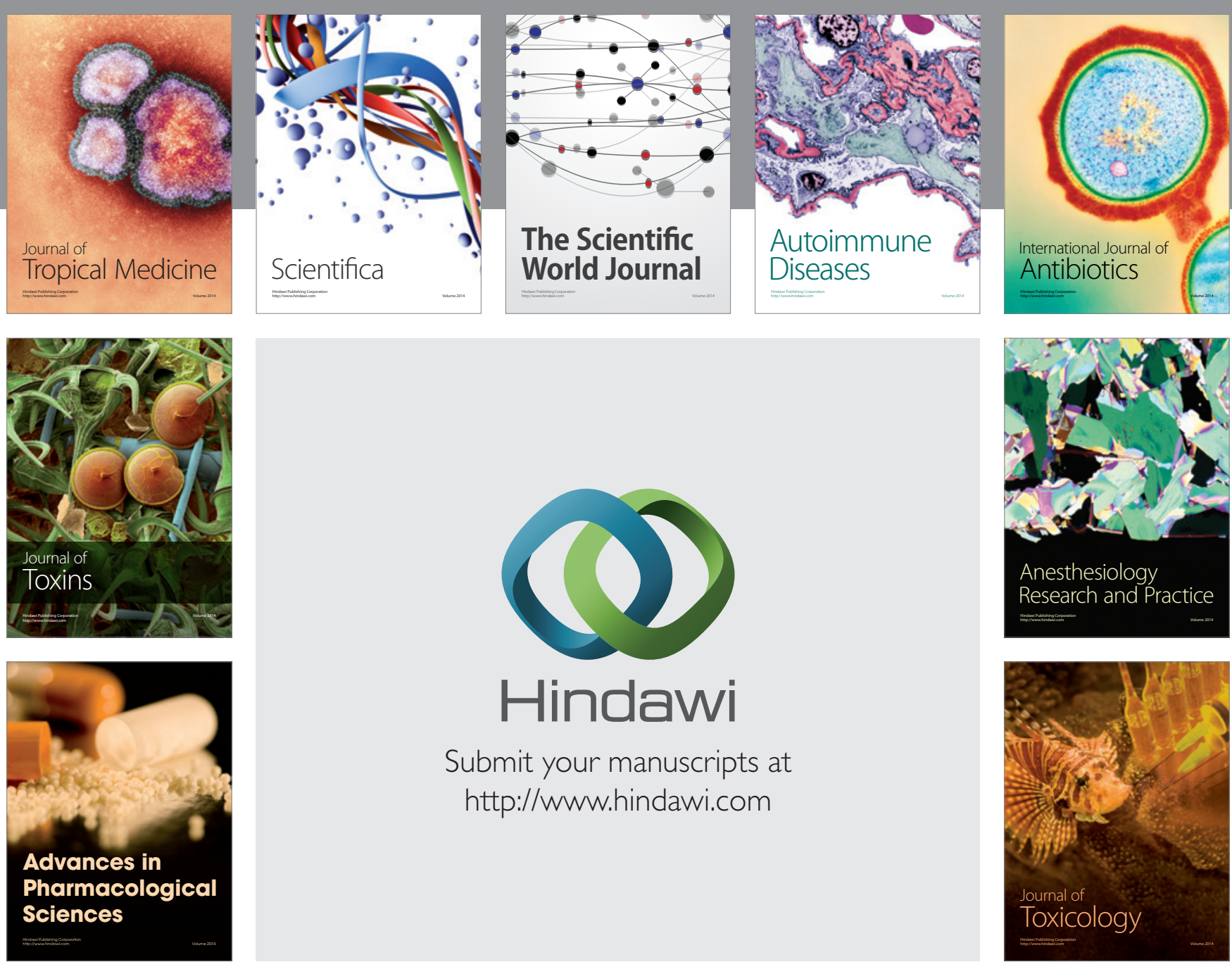

\section{Hindawi}

Submit your manuscripts at

http://www.hindawi.com
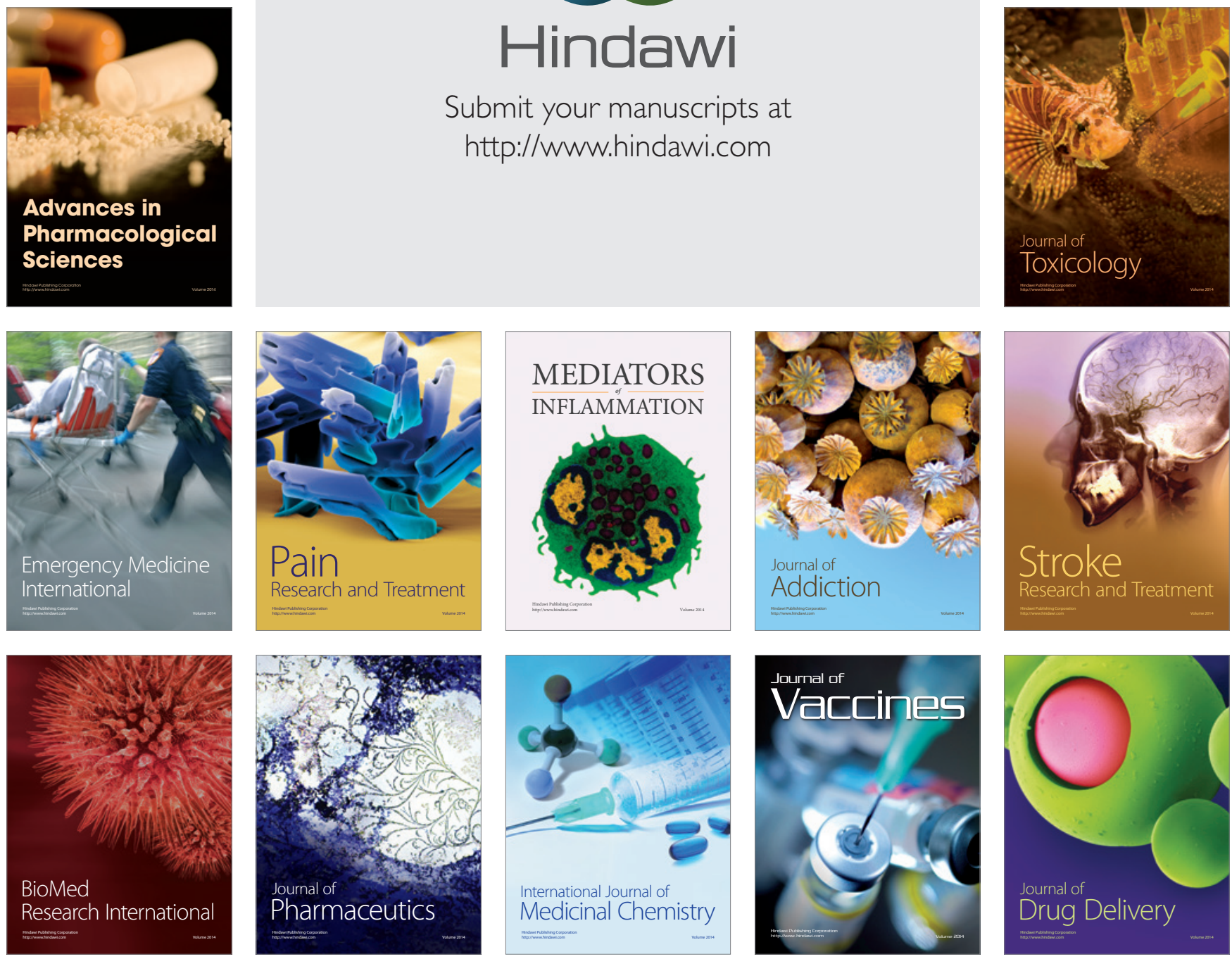